ASI-TPA/8/95

to appear in Reports on Mathematical Physics

\title{
Symmetry, Local Linearization, and Gauge Classification of the Doebner-Goldin Equation
}

\author{
P. Nattermann \\ Institute for Theoretical Physics A \\ Technical University Clausthal \\ D-38678 Clausthal-Zellerfeld, Germany \\ E-mail: aspn@pta3.pt.tu-clausthal.de
}

\begin{abstract}
For the family of nonlinear SCHRÖDINGER equations derived by H.-D. DoEBnER and G.A. Goldin (J. Phys. A 27, 1771) we calculate the complete set of LiE symmetries. For various subfamilies we find different finite and infinite dimensional LIE symmetry algebras. Two of the latter lead to a local transformation linearizing the particular subfamily. One type of these transformations leaves the whole family of equations invariant, giving rise to a gauge classification of the family. The LIE symmetry algebras and their corresponding subalgebras are finally characterized by gauge invariant parameters.
\end{abstract}

\section{Introduction}

In a series of articles [1]- [4] H.-D. DoEBner and G.A. Goldin derived a family of nonlinear Schrödinger equations on $\mathbb{R}^{n}$ from the representation theory of the semi-direct product of the group Diff( $\left(\mathbb{R}^{n}\right)$ of diffeomorphisms on $\mathbb{R}^{n}$ and the ABELian group $C^{\infty}\left(\mathbb{R}^{n}\right)$ of smooth functions on $\mathbb{R}^{n}$, viewed as the 'kinematical symmetry group' of the configuration space $\mathbb{R}^{n}$. Their basic observation is that unitarily inequivalent representations of its LIE algebra $\mathcal{S}\left(\mathbb{R}^{n}\right)=$ $\mathfrak{X}\left(\mathbb{R}^{n}\right) \notin C^{\infty}\left(\mathbb{R}^{n}\right)$ are labeled by a real number $c$, which they interpreted as a new quantum number. On $\mathcal{H}=L^{2}\left(\mathbb{R}^{n}, d^{n} x\right)$ the representatives $\boldsymbol{Q}(f)$ of functions $f \in C^{\infty}\left(\mathbb{R}^{n}\right)$ and $\boldsymbol{P}^{c}(\vec{g})$ of vector-fields $\vec{g} \in \mathfrak{X}\left(I^{n}\right)$ are

$$
\begin{aligned}
(\boldsymbol{Q}(f) \psi)(\vec{x}) & =f(\vec{x}) \psi(\vec{x}), \\
\left(\boldsymbol{P}^{c}(\vec{g}) \psi\right)(\vec{x}) & =\frac{\hbar}{i} \vec{g}(\vec{x}) \cdot(\vec{\nabla} \psi)(\vec{x})+\left(\frac{\hbar}{2 i}+c\right)(\vec{\nabla} \cdot \vec{g})(\vec{x}) \psi(\vec{x}) .
\end{aligned}
$$


A connection between these representations of the kinematical algebra $\mathcal{S}\left(\mathbb{R}^{n}\right)$ and a consistent time evolution of the quantum state can be established as follows [5, 6]1: The representation of $\mathcal{S}\left(\mathbb{R}^{n}\right)$ by functions on the classical phase space $\mathbb{R}^{n} \times \mathbb{R}^{n}$,

$$
\begin{aligned}
Q_{f}(\vec{x}, \vec{p}) & =f(\vec{x}), \\
P_{\vec{g}}(\vec{x}, \vec{p}) & =\vec{p} \cdot \vec{g}(\vec{x}),
\end{aligned}
$$

for a classical system with symmetric and invertible mass matrix $M$, fulfills the dynamical relation

$$
\frac{d}{d t} Q_{f}(\vec{x}(t), \vec{p}(t))=P_{M^{-1} \vec{\nabla} f}(\vec{x}(t), \vec{p}(t)) .
$$

In analogy to this equations a natural requirement for the time evolution of a (mixed) quantum state $\boldsymbol{W}(t) \in \mathcal{T}_{+}^{1}(\mathcal{H})$ is a kind of generalized first EHRENFEST relation

$$
\frac{d}{d t} \operatorname{Tr}(\boldsymbol{W}(t) \boldsymbol{Q}(f))=\operatorname{Tr}\left(\boldsymbol{W}(t) \boldsymbol{P}^{c}\left(M^{-1} \vec{\nabla} f\right)\right)
$$

While there are evolution equations of LINDBLAD type [7] for mixed states fulfilling this equation, we restrict ourselves here to time evolutions of pure states, i.e. we impose the additional constraint $\boldsymbol{W}(t)=|\psi(t)\rangle\langle\psi(t)|$. For a single particle with mass $m, M=m \mathbb{1}$, equation (团) leads to an evolution equation of FOKKER-PLANCK type for the quantum mechanical probability and current densities $\rho=\psi \bar{\psi}$ and $\vec{j}=\frac{\hbar}{2 i m}(\bar{\psi} \vec{\nabla} \psi-\psi \vec{\nabla} \bar{\psi})$ :

$$
\partial_{t} \rho=-\vec{\nabla} \cdot \vec{j}+D \Delta \rho
$$

where $D:=\frac{c}{m}$ is a diffusion parameter. The evolution equation of the wave function $\psi \in$ $L^{2}\left(R^{n}, d^{n} x\right)$ is restricted to a nonlinear Schrödinger equation

$$
i \hbar \partial_{t} \psi=\boldsymbol{H} \psi+i \frac{1}{2} \hbar D \frac{\Delta \rho}{\rho} \psi+R[\psi] \psi
$$

where $\boldsymbol{H}=-\frac{\hbar^{2}}{2 m} \Delta+V$ is a HAMILTON operator and $R[\psi]$ an arbitrary real-valued functional of $\psi$. Requiring for the real functional $R[\psi]$ some of the properties of the imaginary functional $i \frac{1}{2} \hbar D \frac{\Delta \rho}{\rho}$ finally leads to a five parameter family of nonlinear ScHRÖDINGER equations, the general DoEBNER-GoLdiN equation in $n+1$ dimensions:

$$
i \hbar \partial_{t} \psi=\boldsymbol{H} \psi+i \frac{1}{2} \hbar D \frac{\Delta \rho}{\rho} \psi+\hbar D \sum_{j=1}^{5} c_{j} R_{j}[\psi] \psi .
$$

Here the nonlinear functionals $R_{j}[\psi]$ are complex homogeneous of degree zero,

$$
\begin{gathered}
R_{1}[\psi]:=\frac{m}{\hbar} \frac{\vec{\nabla} \cdot \vec{j}}{\rho}, \quad R_{2}[\psi]:=\frac{\Delta \rho}{\rho}, \quad R_{3}[\psi]:=\frac{m^{2}}{\hbar^{2}} \frac{\vec{j}^{2}}{\rho^{2}}, \\
R_{4}[\psi]:=\frac{m}{\hbar} \frac{\vec{j} \cdot \vec{\nabla} \rho}{\rho^{2}}, \quad R_{5}[\psi]:=\frac{(\vec{\nabla} \rho)^{2}}{\rho^{2}}
\end{gathered}
$$

\footnotetext{
${ }^{1}$ The representations and the derivation can be generalized to smooth configuration manifolds.
} 
and $c_{j} \in I R$ are dimensionless 'model' parameters. For the purpose of this paper we will rewrite equation (7) completely in terms of the real functionals $R_{j}[\psi]$, using the decomposition of the LAPLACE operator $\Delta \psi=\left(i R_{1}[\psi]+(1 / 2) R_{2}[\psi]-R_{3}[\psi]-(1 / 4) R_{5}[\psi]\right) \psi$,

$$
i \partial_{t} \psi=i \sum_{j=1}^{2} \nu_{j} R_{j}[\psi] \psi+\sum_{j=1}^{5} \mu_{j} R_{j}[\psi] \psi+\mu_{0} V \psi, \quad \nu_{1} \neq 0
$$

Homogeneous nonlinear SCHRÖDINGER equations of this type, though with a different choice of non-vanishing complex parameters $\mu_{j}+i \nu_{j}$, were introduced in [16]. In our case the free equation $(V \equiv 0)$ can be viewed as a seven parameter family of nonlinear Schrödinger equations.

Several properties of the DG-equation have already been observed such as its homogeneity and separability, the linearization and НАміLтоNian formulation of a subfamily, and stationary as well as some non-stationary solutions have been found [2-5,8-15]. Nevertheless, apart from the invariance of a subfamily under GALILEI transformations, the LIE symmetry of this partial differential equation (PDE) was only investigated for small nonlinearities of the $1+1$ dimensional DG-equation [17].

In the case of the free linear SCHRÖDINGER equation (SE) the LIE symmetry group is well known and amounts to a projective unitary representation of the 'SCHRÖDINGER group' 18, 19, 20

$$
S \operatorname{ch}(n):=(S L(2, I R) \otimes S O(n)) \otimes(T(n) \otimes T(n)),
$$

where the action of $S O(n)$ on the ABELian groups $T(n)$ (representing space translations and special GALILEI transformations) is as usual and the elements of $S L(2, I R)$ act on $T(n) \otimes T(n)$ as $2 \times 2$ matrices. Due to the linearity of the SE there are two additional types of symmetry transformations: on the one hand the multiplication of the wavefunction by a real number (real homogeneity)f, on the other hand the addition of an arbitrary solution of the equation, which is in fact an infinite dimensional symmetry group.

In section 2 we determine the maximal LIE symmetry algebra of the free $(V \equiv 0) \mathrm{DG}^{-}$ equation. Exploiting the condition for a generator of a LIE symmetry in section 2.1 we are led to distinguish various subfamilies. Among the LIE symmetries there are finite and an infinite dimensional ones and they will be discussed separately in sections 2.2 and 2.3. It turns out that part of the infinite dimensional symmetry corresponds to a linearization of a subfamily of the DG-equation [8, 9]. As the symmetry algebra is maximal the linearizing transformations obtained here are the only local ones, i.e. the only ones depending purely on the values of the wave functions.

One type of these transformations also leaves the probability density invariant and has therefore been called nonlinear gauge transformation [10. As we will see in section 3 the whole family of DG-equations is invariant under these gauge transformations. Summarizing the results we are finally led to a gauge invariant classification of the family (7) and its symmetries.

\footnotetext{
${ }^{2}$ Note that the multiplication with a phase factor is already contained in the projective representation of $\operatorname{Sch}(n)$.
} 


\section{Lie symmetries}

\subsection{Determining equations}

The methods of finding LIE symmetry algebras of PDEs have been discussed in detail in the monographs [21, 22, 23] and computer algebra programs are available for the calculations, e.g. [24]. Here we sketch roughly the procedure to fix our notation.

Using the polar decomposition for the wavefunction $\psi(\vec{x}, t)=\exp [r(\vec{x}, t)+i s(\vec{x}, t)]$, the free $(V \equiv 0)$ complex evolution equation (9) leads to two real evolution equations for the real functions $r$ and $s$ :

$$
\begin{aligned}
\mathfrak{F}_{1}\left(r_{t}, \vec{\nabla} r, \Delta r, s_{t}, \vec{\nabla} s, \Delta s\right):= & -r_{t}+2 \nu_{2} \Delta r+\nu_{1} \Delta s+4 \nu_{2}(\vec{\nabla} r)^{2}+2 \nu_{1} \vec{\nabla} r \cdot \vec{\nabla} s \\
= & 0, \\
\mathfrak{F}_{2}\left(r_{t}, \vec{\nabla} r, \Delta r, s_{t}, \vec{\nabla} s, \Delta s\right):= & s_{t}+2 \mu_{2} \Delta r+\mu_{1} \Delta s+4\left(\mu_{2}+\mu_{5}\right)(\vec{\nabla} r)^{2} \\
& +2\left(\mu_{1}+\mu_{4}\right) \vec{\nabla} r \cdot \vec{\nabla} s+\mu_{3}(\vec{\nabla} s)^{2} \\
= & 0,
\end{aligned}
$$

where we have used the usual indication of partial derivatives by subscripts.

A sufficient condition for the vector-field

$$
X=\sum_{j=1}^{n} \xi^{(j)}(\vec{x}, t, r, s) \partial_{x_{j}}+\tau(\vec{x}, t, r, s) \partial_{t}+\phi(\vec{x}, t, r, s) \partial_{r}+\sigma(\vec{x}, t, r, s) \partial_{s}
$$

on the space of independent $(\vec{x}, t)$ and dependent variables $(r, s)$ to be a generator of a symmetry group of transformations of the system (11) described by the map $\mathfrak{F}=\left(\mathfrak{F}_{1}, \mathfrak{F}_{2}\right)$ is the vanishing action of its second prolongation $\operatorname{pr}^{(2)} X$ on $\mathfrak{F}$ restricted to its solution variety $\Sigma:=\mathfrak{F}^{-1}(0)$,

$$
\left(\operatorname{pr}^{(2)} X \mathfrak{F}_{j}\right)_{\left.\right|_{\Sigma}}=0, \quad j=1,2 .
$$

For the system (11) this condition is also necessary since $\mathfrak{F}$ is analytic and of rank 2 on $\Sigma$ [22, Theorem 2.71, Lemma 2.74]. Thus, the calculation of all vector-fields $X$ fulfilling (13) yields its maximal LIE symmetry algebra.

Condition (13) leads to a set of linear PDEs called determining equations for the coefficients $\xi^{(j)}, \tau, \phi$, and $\sigma$ of the vector-field $X$. Since $\mathfrak{F}$ depends only on the dependent variables $(r, s)$, their first order derivatives, and their Laplacian, calculations are reduced considerably. A set of trivial equations among the determining equations yield $\xi^{(j)}=\xi^{(j)}(\vec{x}, t)$ and $\tau=\tau(t)$. With this restricted dependence the remaining independent determining equations are

$$
\begin{aligned}
-\xi_{t}^{(j)}-\mu_{1} \Delta \xi^{(j)}+2\left(\mu_{1}+\mu_{4}\right) \phi_{x_{j}}+4 \mu_{2} \phi_{x_{j} s}+2 \mu_{3} \sigma_{x_{j}}+2 \mu_{1} \sigma_{x_{j} s} & =0, \\
-\nu_{1} \Delta \xi^{(j)}+2 \nu_{1} \phi_{x_{j}}+4 \nu_{2} \phi_{x_{j} s}+2 \nu_{1} \sigma_{x_{j} s} & =0, \\
-\mu_{2} \Delta \xi^{(j)}+4\left(\mu_{2}+\mu_{5}\right) \phi_{x_{j}}+2 \mu_{2} \phi_{x_{j} r}+\left(\mu_{1}+\mu_{4}\right) \sigma_{x_{j}}+\mu_{1} \sigma_{x_{j} r} & =0, \\
\xi_{t}^{(j)}-2 \nu_{2} \Delta \xi^{(j)}+8 \nu_{2} \phi_{x_{j}}+4 \nu_{2} \phi_{x_{j} r}+2 \nu_{1} \sigma_{x_{j}}+2 \nu_{1} \sigma_{x_{j} r} & =0,
\end{aligned}
$$




$$
\begin{aligned}
\sigma_{t}+\mu_{1} \Delta \sigma+2 \mu_{2} \Delta \phi & =0, \\
-\phi_{t}+2 \nu_{2} \Delta \phi+\nu_{1} \Delta \sigma & =0, \\
\xi_{x_{k}}^{(j)}+\xi_{x_{j}}^{(k)} & =0, \\
2\left(\mu_{1}+\mu_{4}\right) \phi_{s}+2 \mu_{2} \phi_{s s}+\mu_{3} \sigma_{s}+\mu_{1} \sigma_{s s}+\mu_{3} \tau_{t}-2 \mu_{3} \xi_{x_{j}}^{(j)} & =0, \\
2 \mu_{2} \phi_{s}+\nu_{1} \sigma_{r}+\mu_{1} \tau_{t}-2 \mu_{1} \xi_{x_{j}}^{(j)} & =0, \\
\left(\mu_{1}+2 \nu_{2}\right) \phi_{s}-\nu_{1} \phi_{r}+\nu_{1} \sigma_{s}+\nu_{1} \tau_{t}-2 \nu_{1} \xi_{x_{j}}^{(j)} & =0, \\
4\left(\mu_{2}+\mu_{5}\right) \phi_{s}+\left(\mu_{1}+\mu_{4}\right) \phi_{r}+2 \mu_{2} \phi_{r s} & \\
+\left(\mu_{3}+\nu_{1}\right) \sigma_{r}+\mu_{1} \sigma_{r s}+\left(\mu_{1}+\mu_{4}\right) \tau_{t}-2\left(\mu_{1}+\mu_{4}\right) \xi_{x_{j}}^{(j)} & =0, \\
2 \mu_{2} \phi_{r}-2 \mu_{2} \sigma_{s}+\left(\mu_{1}+2 \nu_{2}\right) \sigma_{r}+2 \mu_{2} \tau_{t}-4 \mu_{2} \xi_{x_{j}}^{(j)} & =0, \\
2 \mu_{2} \phi_{s}+\nu_{1} \sigma_{r}+2 \nu_{2} \tau_{t}-4 \nu_{2} \xi_{x_{j}}^{(j)} & =0, \\
8\left(\mu_{2}+\mu_{5}\right) \phi_{r}-4\left(\mu_{2}+\mu_{5}\right) \sigma_{s} & \\
\left.+\mu_{1}+\mu_{4}+4 \nu_{2}\right) \phi_{s}+2 \nu_{2} \phi_{r s}+\nu_{1} \sigma_{s}+\nu_{1} \sigma_{r s}+\nu_{1} \tau_{t}-2 \nu_{1} \xi_{x_{j}}^{(j)} & =0, \\
4\left(\mu_{2}+\mu_{5}\right) \phi_{s}+4 \nu_{2} \phi_{r}+2 \nu_{2} \phi_{r r}+2 \nu_{1} \sigma_{r}+\nu_{1} \sigma_{r r}+4 \nu_{2} \tau_{t}-8 \nu_{2} \xi_{x_{j}}^{(j)} & =0, \\
\mu_{3} \phi_{s}+2 \nu_{1} \phi_{s}+2 \nu_{2} \phi_{s s}+\nu_{1} \sigma_{s s} & =0 .
\end{aligned}
$$

Combining the derivative of equation (21) with respect to $s$ with equation (30) we obtain a linear second order differential equation for $\phi_{s}$ as a function of $s$,

$$
\alpha_{1} \phi_{s s s}+\alpha_{2} \phi_{s s}+\alpha_{3} \phi_{s}=0
$$

with constant coefficients $\alpha_{1}=2\left(\nu_{1} \mu_{2}-\nu_{2} \mu_{1}\right), \alpha_{2}=2 \nu_{1} \mu_{4}-\mu_{3}\left(\mu_{1}+2 \nu_{2}\right)$, and $\alpha_{3}=-\mu_{3}\left(\mu_{3}+\right.$ $2 \nu_{1}$ ). Another ordinary differential equation of this type is obtained from equations (23), (26), (30), and their differential consequences:

$$
\beta_{1} \phi_{s s}+\beta_{2} \phi_{s}=0
$$

where $\beta_{1}=\nu_{1}\left(\mu_{4}+2 \nu_{2}\right)-\mu_{1}\left(\mu_{3}+\nu_{1}\right)$ and $\beta_{2}=\left(\mu_{3}+2 \nu_{1}\right)\left(\mu_{3}+\nu_{1}\right)$.

Thus, for the general $s$-dependence of $\phi$ we have to distinguish four different cases. First we have a polynomial dependence of $\phi$ on $s$ of maximal order two, if at most one of the $\alpha_{j}$ and one of the $\beta_{j}$ is non-vanishing. Then there are exponential solutions of (31) and (32) (including oscillating solutions), as well as aperiodic solutions of (31). In all these cases the $s$-dependence of $\sigma$ is obtained by an integration of (30). Finally, if all coefficients vanish, $\alpha_{j}=\beta_{k}=0$, there is no information on the $s$-dependence of $\phi$ and $\sigma$, but instead, using equation (24) as well, we get a restriction on the parameters:

$$
\mu_{2}=\frac{\nu_{2} \mu_{1}}{\nu_{1}}, \quad \mu_{3}=-2 \nu_{1}, \quad \mu_{4}=-2 \nu_{2}-\mu_{1}, \quad \mu_{5}=-\frac{\nu_{2} \mu_{1}}{\nu_{1}}
$$


Since the solutions and their derivatives in the different cases are functionally independent and the determining equations are linear we can investigate them independently. Calculations for the different cases show that in general we have two kinds of symmetries, a set of finite dimensional LIE symmetries arising from polynomial or exponential solutions, and a set of infinite dimensional LIE symmetries connected with exponential solutions or the subfamily (33). The aperiodic solution turns out to be consistent only for this subfamily as well.

We will discuss the finite and infinite symmetries separately in the next two sections.

\section{$2.2 \quad$ Finite symmetries}

Let us begin with the polynomial solutions of (31) and (32), i.e.

$$
\phi(\vec{x}, t, r, s)=\phi^{(2)}(\vec{x}, t, r) s^{2}+\phi^{(1)}(\vec{x}, t, r) s+\phi^{(0)}(\vec{x}, t, r),
$$

in which case $\sigma$ integrates to

$$
\begin{aligned}
\sigma(\vec{x}, t, r, s)= & -\frac{\mu_{3}+2 \nu_{1}}{3 \nu_{1}} \phi^{(2)}(\vec{x}, t, r) s^{3}-\left(\frac{\mu_{3}+2 \nu_{1}}{2 \nu_{1}} \phi^{(1)}(\vec{x}, t, r)+\frac{2 \nu_{2}}{\nu_{1}} \phi^{(2)}(\vec{x}, t, r)\right) s^{2} \\
& +\left(\sigma^{(1)}(\vec{x}, t, r)-\frac{\mu_{3}+2 \nu_{1}}{\nu_{1}} \phi^{(0)}(\vec{x}, t, r)-\frac{2 \nu_{2}}{\nu_{1}} \phi^{(1)}(\vec{x}, t, r)\right) s+\sigma^{(0)}(\vec{x}, t, r)
\end{aligned}
$$

Neglecting the special case (33), which will be treated in the next section, we obtain from equations (22)-(29)

$$
\phi^{(1)} \equiv \phi^{(2)} \equiv \phi_{r}^{(0)} \equiv \sigma_{r}^{(1)} \equiv 0 \quad \text { and } \quad \sigma_{r}^{(0)} \equiv-\frac{2 \nu_{2}}{\nu_{1}}\left(\tau_{t}-2 \xi_{x_{j}}^{(j)}\right)
$$

With these results equations (14)-(20) lead to a polynomial dependence of the coefficients of the vector-field $X$ on all variables:

$$
\begin{aligned}
\vec{\xi}(\vec{x}, t) & =k_{1} t \vec{x}+k_{2} \vec{x}+A \vec{x}+\vec{b} t+\vec{a}, \\
\tau(t) & =k_{1} t^{2}+\left(2 k_{2}-k_{6}\right) t+k_{3}, \\
\phi(t, f) & =-\frac{n}{2} k_{1} t+k_{4}, \\
\sigma(\vec{x}, t) & =\frac{2 \nu_{2}}{\nu_{1}} k_{6} r+k_{6} s-\frac{1}{4 \nu_{1}} k_{1} \vec{x}^{2}-\frac{1}{2 \nu_{1}} \vec{b} \cdot \vec{x}+\frac{n \mu_{1}}{2 \nu_{1}} k_{1} t+k_{5}-\frac{2 \nu_{2}}{\nu_{1}} k_{4},
\end{aligned}
$$

where $A$ is a real antisymmetric matrix and $k_{j}, \vec{a}, \vec{b}$ are real constants with the additional conditions

$$
\begin{aligned}
k_{1}=0, \vec{b}=\overrightarrow{0} \quad \text { or } \quad \mu_{1}+\mu_{4}=\mu_{3}+\nu_{1}=0, \\
k_{6}=0 \quad \text { or } \quad \mu_{1}=2 \nu_{2}, \quad \mu_{2}=\frac{2 \nu_{2}^{2}}{\nu_{1}}, \quad \mu_{4}=\frac{2 \mu_{3} \nu_{2}}{\nu_{1}}, \quad \mu_{5}=\frac{\mu_{3} \nu_{2}^{2}}{\nu_{1}^{2}} .
\end{aligned}
$$

For the LIE algebra spanned by these vector-fields we choose the following basis $(j, k=$ $1, \ldots, n)$ :

$$
\begin{gathered}
L_{j k}=x_{j} \partial_{x_{k}}-x_{k} \partial_{x_{j}}, \quad H=\partial_{t}, \quad D=\sum_{j=1}^{n} x_{j} \partial_{x_{j}}+2 t \partial_{t}-\frac{n}{2} \partial_{r}+\frac{n \mu_{1}}{2 \nu_{1}} \partial_{s}, \\
C=\sum_{j=1}^{n} x_{j} t \partial_{x_{j}}+t^{2} \partial_{t}-\frac{n}{2} t \partial_{r}-\left(\frac{1}{4 \nu_{1}} \vec{x}^{2}-\frac{n \mu_{1}}{2 \nu_{1}} t\right) \partial_{s}, \quad P_{j}=\partial_{x_{j}} \\
B_{j}=t \partial_{x_{j}}-\frac{1}{2 \nu_{1}} x_{j} \partial_{s}, \quad E=-\frac{1}{2 \nu_{1}} \partial_{s}, \quad R=\partial_{r}, \quad A=-t \partial_{t}+\left(\frac{2 \nu_{2}}{\nu_{1}} r+s\right) \partial_{s} .
\end{gathered}
$$


This leads to the following nontrivial commutators $(j, k, l, m=1, \ldots, n)$ :

$$
\begin{gathered}
{[D, H]=-2 H, \quad[H, C]=D, \quad[D, C]=2 C, \quad\left[H, B_{j}\right]=P_{j},} \\
{\left[D, P_{j}\right]=-P_{j}, \quad\left[D, B_{j}\right]=B_{j}, \quad\left[C, P_{j}\right]=-B_{j}, \quad\left[P_{j}, B_{k}\right]=\delta_{j k} E,} \\
{[A, H]=H, \quad[A, C]=-C, \quad[A, E]=-E, \quad[A, R]=4 \nu_{2} E, \quad\left[A, B_{j}\right]=-B_{j},} \\
{\left[L_{j k}, P_{l}\right]=\delta_{k l} P_{j}-\delta_{j l} P_{k}, \quad\left[L_{j k}, B_{l}\right]=\delta_{k l} B_{j}-\delta_{j l} B_{k},} \\
{\left[L_{j k}, L_{l m}\right]=\delta_{k l} L_{j m}+\delta_{j m} L_{k l}-\delta_{j l} L_{k m}-\delta_{k m} L_{j l} .}
\end{gathered}
$$

Now if both conditions (38) and (39) are fulfilled, i.e. for the two parameter subfamily

$$
\mu_{1}=2 \nu_{2}, \quad \mu_{2}=\frac{2 \nu_{2}^{2}}{\nu_{1}}, \quad \mu_{3}=-\nu_{1}, \quad \mu_{4}=-2 \nu_{2}, \quad \mu_{5}=-\frac{\nu_{2}^{2}}{\nu_{1}}
$$

we get an $\frac{(n+1)(n+2)}{2}+5$ dimensional LIE symmetry algebra of the DG-equation (9). $\{H, D, C\}$ generate the subalgebra $s l(2, I R),\left\{P_{j}\right\}$ and $\left\{B_{j}\right\}$ two $n$-dimensional commutative subalgebras, and $\left\{L_{j k}\right\}$ the subalgebra $s o(n)$. Together these generators span the ScHRöDINGER algebra $\operatorname{sch}(n)$. Note, however, that the representation of $\operatorname{sch}(n)$ differs from the one obtained for the linear SE [18, 19] by those terms proportional to $\mu_{1}$ in $D$ and $C$; the reason will be explained in the section 3. Furthermore, the generator $R+4 \nu_{2} E$ is easily seen to be independent of the rest, and $\{A, E\}$ span a one dimensional affine algebra that acts on the $\operatorname{sl}(2, I R)$ - and the boost-subalgebra of the SCHRÖDINGER algebra $\operatorname{sch}(n)$. Hence, the structure of this LIE symmetry can be written as

$$
\operatorname{sym}_{3}(n)=(\operatorname{aff}(1) \oplus \operatorname{sch}(n)) \oplus t(1) .
$$

If only (38) is fulfilled, $A$ is not a generator of a LIE symmetry of (9), and $E$ becomes the centre of the extension $\operatorname{sch}_{e}(n)$ of the SCHRÖDINGER algebra. Thus, we obtain for the five parameter subfamily (38) the finite dimensional symmetry algebra

$$
\operatorname{sym}_{1}(n)=\operatorname{sch}_{e}(n) \oplus t(1) .
$$

It is exactly the finite dimensional part of the maximal LIE symmetry algebra of the linear SE including real homogeneity but excluding the (infinite dimensional) part due to the additivity of the linear SE.

If on the other hand condition (38) is not fulfilled, but (39) is, $C$ and $B_{j}$ are no longer generators of a LIE symmetry, the SCHRÖDINGER subalgebra of $\operatorname{sym}_{3}(n)$ is reduced to a direct sum of aff (1) and the EUCLIDean algebra $e(n)$, and we obtain as the LIE symmetry algebra for the three parameter subfamily (39):

$$
\operatorname{sym}_{2}(n)=(\operatorname{aff}(1) \oplus(\operatorname{aff}(1) \oplus e(n))) \oplus t(1) .
$$

However, if we impose no conditions at all, the LIE symmetry algebra is restricted to the intersection of $\operatorname{sym}_{1}(n)$ and $\operatorname{sym}_{2}(n)$ :

$$
\operatorname{sym}_{0}(n)=(\operatorname{aff}(1) \notin e(n)) \oplus t(2) .
$$


As mentioned previously the exponential solution of (31) and (32),

$$
\begin{aligned}
\phi(\vec{x}, t, r, s) & =\phi^{(1)}(\vec{x}, t, r) e^{\lambda s}, \\
\sigma(\vec{x}, t, r, s) & =-\left(\frac{\mu_{3}+2 \nu_{1}}{\nu_{1}} \lambda^{-1}+\frac{2 \nu_{2}}{\nu_{1}}\right) \phi^{(1)}(\vec{x}, t, r) e^{\lambda s},
\end{aligned}
$$

also leads to a finite dimensional LIE symmetry. The determining equation (23) implies that the $r$-dependence is also exponential,

$$
\phi^{(1)}(\vec{x}, t, r)=\phi^{(2)}(\vec{x}, t) e^{\eta r}, \quad \eta=\frac{\mu_{1}}{\nu_{1}} \lambda-\frac{\mu_{3}+2 \nu_{1}}{\nu_{1}} .
$$

The remaining determining equations are consistent with this solution in three particular cases. One of them leads to the subfamily (33), another to an infinite dimensional LIE symmetry; both will be treated in the next section. The third case implies $\lambda=2 \frac{\mu_{3}+\nu_{1}}{\mu_{1}-2 \nu_{2}}$ and $\phi^{(2)}=$ const, and restricts the parameters to

$$
\begin{gathered}
\mu_{2}=\frac{\mu_{3}\left(\mu_{1}+2 \nu_{2}\right)^{2}\left(\mu_{3}+2 \nu_{1}\right)+8 \mu_{1} \nu_{1}^{2} \nu_{2}}{8 \nu_{1}\left(\mu_{3}+\nu_{1}\right)^{2}}, \quad \mu_{4}=\frac{\mu_{3}\left(\mu_{1}+2 \nu_{2}\right)}{2 \nu_{1}}, \quad \mu_{5}=\frac{\mu_{3}}{2 \nu_{1}} \mu_{2}, \\
\mu_{1}-2 \nu_{2} \neq 0 \neq \mu_{3}+\nu_{1} .
\end{gathered}
$$

Obviously these conditions are not consistent with (38) and (39), so we get the generator

$$
F=e^{\eta r+\lambda s}\left(\partial_{r}-\kappa \partial_{s}\right), \quad \kappa=\frac{\mu_{3}\left(\mu_{1}+2 \nu_{2}\right)+2 \nu_{1} \nu_{2}}{2 \nu_{1}\left(\nu_{1}+\mu_{3}\right)},
$$

only in addition to generators of the LIE symmetry $\operatorname{sym} m_{0}(n)$ (46). If we replace $D$ by $D^{\prime}=$ $\sum_{j=1}^{n} x_{j} \partial_{x_{j}}+2 t \partial_{t}$ the additional nontrivial commutation relations are

$$
[F, E]=\frac{\lambda}{2 \nu_{1}} F, \quad[F, R]=-\eta F
$$

Thus, $\{F, E, R\}$ span a subalgebra and we obtain as the maximal LIE algebra in this case:

$$
\operatorname{sym}_{4}(n)=(t(2) \notin t(1)) \oplus(\operatorname{aff}(1) \oplus e(n)) .
$$

The one parameter groups of transformations $\Phi_{\varepsilon}^{X}$ of the wavefunction corresponding to the vector-fields $L_{j k}, P_{j}, B_{j}, H_{j}, E$, and $R$ are well known from the linear SE, so we only give them for the new generators $A$ and $F$ and the slightly modified $D$ and $C$ :

$$
\begin{aligned}
\left(\Phi_{\varepsilon}^{D} \psi\right)(\vec{x}, t)= & \exp \left(-\varepsilon \frac{n}{2}+i \varepsilon \frac{n \mu_{1}}{2 \nu_{1}}\right) \psi\left(\vec{x} e^{-\varepsilon}, t e^{-2 \varepsilon}\right), \\
\left(\Phi_{\varepsilon}^{C} \psi\right)(\vec{x}, t)= & (1-\varepsilon t)^{-\frac{n}{2}} \exp \left(i\left(\frac{\varepsilon}{2 \nu_{1}(1-\varepsilon t)} \vec{x}^{2}+\frac{n \mu_{1}}{\nu_{1}} \ln |1-\varepsilon t|\right)\right) \psi\left(\frac{\vec{x}}{1-\varepsilon t}, \frac{t}{1-\varepsilon t}\right), \\
\left(\Phi_{\varepsilon}^{A} \psi\right)(\vec{x}, t)= & \exp \left(i\left(\left(e^{\varepsilon}-1\right)\left(\frac{2 \nu_{2}}{\nu_{1}} \ln \left|\psi\left(\vec{x}, e^{\varepsilon} t\right)\right|+\arg \psi\left(\vec{x}, e^{\varepsilon} t\right)\right)\right)\right) \psi\left(\vec{x}, e^{\varepsilon} t\right), \\
\left(\Phi_{\varepsilon}^{F} \psi\right)(\vec{x}, t)= & \sqrt{2 \varepsilon+e^{-\lambda \arg \psi(\vec{x}, t)}|\psi(\vec{x}, t)|^{-\eta}}|\psi(\vec{x}, t)|^{\frac{\eta}{2}} \exp \left(\frac{\lambda}{2} \arg \psi(\vec{x}, t)\right. \\
& -i \frac{\kappa}{2}\left(\ln \left(2 \varepsilon+e^{-\lambda \arg \psi(\vec{x}, t)}|\psi(\vec{x}, t)|^{-\eta}\right)+\lambda \arg \psi(\vec{x}, t)\right. \\
& +\eta \ln |\psi(\vec{x}, t)|)) \psi(\vec{x}, t) .
\end{aligned}
$$

The transformations generated by $C$ are called projective [25] or conformal [19] transformations or expansions [18], whereas $D$ generates a scaling of the independent variables $(\vec{x}, t)$ called dilations. 


\section{$2.3 \quad$ Infinite symmetries}

As indicated in section 2.1 there are two cases in which we obtain an additional infinite dimensional LIE symmetry. Let us treat the case $\alpha_{j}=\beta_{k}=0$ in equations (31), (32) first, i.e. we restrict our calculations to the three parameter subfamily (33). This simplifies the determining equations (14)-(30) considerably. Equations (23), (24), (26), (29), and (30) imply that

$$
\sigma(\vec{x}, t, r, s)=-\frac{2 \nu_{2}}{\nu_{1}} \phi(\vec{x}, t, r, s)+r \sigma^{(1)}(\vec{x}, t)+s \sigma^{(2)}(\vec{x}, t)+\sigma^{(0)}(\vec{x}, t) .
$$

From (14)-(19) we infer furthermore that $\phi$ and $\sigma$ do not depend on $(\vec{x}, t)$ and that $\xi^{(j)}$ and $\tau$ lead to generators of the symmetry $\operatorname{sym}_{0}(n)(46)$, which have been discussed in the previous section. Then (21) and (29) imply that $\sigma^{(1)} \equiv \sigma^{(2)} \equiv 0$, and (23) yields an equation for $\phi^{(1)}$,

$$
\nu_{1} \phi_{r}^{(1)}-\mu_{1} \phi_{s}^{(1)}=0
$$

Integrating this equation and neglecting the generators of complex homogeneity $R$ and $E$, which we have already discussed in the previous section, we obtain the generators of an infinite dimensional LIE symmetry

$$
Y_{f}=f\left(\mu_{1} r+\nu_{1} s\right)\left(\partial_{r}-\frac{2 \nu_{2}}{\nu_{1}} \partial_{s}\right)
$$

If $\mu_{1}=2 \nu_{2}$, the algebra $a^{\infty}=\left\{Y_{f} \mid f \in C^{\infty}(I R)\right\}$ is a representation of the infinite dimensional commutative LIE algebra $C^{\infty}(I R)$. Otherwise it is a representation of the infinite dimensional LIE algebra $\mathfrak{X}(\mathbb{R})$ of vector-fields on $\mathbb{R}$, where the commutator is defined by $\left[f_{1}, f_{2}\right](z):=$ $f_{1}(z) f_{2}^{\prime}(z)-f_{2}(z) f_{1}^{\prime}(z)$ :

$$
\left[Y_{f_{1}}, Y_{f_{2}}\right]=\left(\mu_{1}-2 \nu_{2}\right) Y_{\left[f_{1}, f_{2}\right]} .
$$

In the commutative case condition (33) is compatible with condition (39) of the finite algebra $\operatorname{sym}_{2}(n)$. The nontrivial commutation relations of $Y_{f}$ with the generators of $s y m_{2}(n)$ are

$$
\left[Y_{f}, R\right]=-\mu_{1} Y_{f^{\prime}}, \quad\left[Y_{f}, E\right]=\frac{1}{2} Y_{f^{\prime}}, \quad\left[Y_{f}, A\right]=Y_{z f^{\prime}}
$$

Still, $R+4 \nu_{2} E$ commutes with the rest of the algebra and for the two parameter subfamily

$$
\mu_{1}=2 \nu_{2}, \quad \mu_{2}=2 \frac{\nu_{2}^{2}}{\nu_{1}}, \quad \mu_{3}=-2 \nu_{1}, \quad \mu_{4}=-4 \nu_{2}, \quad \mu_{5}=-2 \frac{\nu_{2}^{2}}{\nu_{1}}
$$

we obtain the maximal LIE algebra

$$
\operatorname{sym}_{2}^{a}(n)=\operatorname{sym}_{2}(n) \oplus a^{\infty}=\left(\operatorname{aff}(1) \oplus\left(\left(a f f(1) \oplus e_{n}(n)\right) \oplus a^{\infty}\right)\right) \oplus t(1) .
$$

The one parameter group of transformations generated by $Y_{f}$ can be calculated explicitly in the commutative case:

$$
\left(\Phi_{\varepsilon}^{D} \psi\right)(\vec{x}, t)=\exp \left(\varepsilon f\left(2 \nu_{2} \ln |\psi(\vec{x}, t)|+\nu_{1} \arg \psi(\vec{x}, t)\right)\left(1-\frac{2 \nu_{2}}{\nu_{1}}\right)\right) \psi(\vec{x}, t)
$$


For the non commutative case $A$ is no longer a generator of a LIE symmetry, and we obtain the maximal LIE symmetry

$$
\operatorname{sym}_{0}^{a}(n)=\operatorname{sym}_{0}(n) \oplus a^{\infty}=(\operatorname{aff}(1) \oplus e(n)) \oplus\left(t(1) \oplus a^{\infty}\right) \oplus t(1)
$$

of the three parameter subfamily (33). Note that the finite dimensional algebra $\operatorname{sym}_{4}(n)$ is contained in $\operatorname{sym}_{0}^{a}(n)$.

Another infinite dimensional LIE symmetry algebra is obtained from the exponential solution (47) and (48). To simplify calculations we can allow $\lambda$ to be complex valued, as the determining equations are linear. We mentioned in the previous section that only in three cases the exponential ansatz is consistent with the determining equations (14)-(30); one case led to a finite symmetry, another leads to the infinite symmetry already discussed in this section. The remaining case requires a restriction to the three parameter subfamily

$$
\mu_{1}=2 \nu_{2}, \quad \mu_{3}=-\nu_{1}, \quad \mu_{4}=-2 \nu_{2}, \quad \mu_{5}=-\frac{1}{2} \mu_{2}, \quad \mu_{2} \neq 2 \frac{\nu_{2}^{2}}{\nu_{1}},
$$

and the coefficient of the exponential function is fixed by

$$
\lambda^{2}=\frac{\nu_{1}^{2}}{4 \nu_{2}^{2}-2 \nu_{1} \mu_{2}} .
$$

So we have to distinguish two cases.

If $\mu_{2}<2 \frac{\nu_{2}^{2}}{\nu_{1}}, \lambda= \pm|\lambda|$ is real and we get the generators

$$
\begin{aligned}
Z_{\Phi_{ \pm}}= & e^{-r}\left(\Phi_{+}(\vec{x}, t) e^{|\lambda|\left(\frac{2 \nu_{2}}{\nu_{1}} r+s\right)}\left(|\lambda| \partial_{r}-\left(1+\frac{2 \nu_{2}}{\nu_{1}}|\lambda|\right) \partial_{s}\right)\right. \\
& \left.+\Phi_{-}(\vec{x}, t) e^{-|\lambda|\left(\frac{2 \nu_{2}}{\nu_{1}} r+s\right)}\left(|\lambda| \partial_{r}+\left(1-\frac{2 \nu_{2}}{\nu_{1}}|\lambda|\right) \partial_{s}\right)\right)
\end{aligned}
$$

where $\Phi_{+}\left(\Phi_{-}\right)$is a (smooth) solution of the forward (backward) heat equation with diffusion coefficient $\sqrt{4 \nu_{2}^{2}-2 \nu_{1} \mu_{2}}$, if $\nu_{1}>0$ (The role of $\Phi_{+}$and $\Phi_{-}$is interchanged if $\nu_{1}<0$ ):

$$
\partial_{t} \Phi_{ \pm} \pm \sqrt{4 \nu_{2}^{2}-2 \nu_{1} \mu_{2}} \Delta \Phi_{ \pm}=0 .
$$

The algebra $b^{\infty}=\left\{Z_{\Phi_{ \pm}} \mid \Phi_{ \pm}\right.$solution of (67) $\}$is commutative, and the subfamily (64) possesses also the finite dimensional symmetry $\operatorname{sym}_{1}(n)$, whose generators act on the generators $Z_{\Phi_{ \pm}}$. Hence, we obtain the full LIE symmetry algebra

$$
\operatorname{sym}_{1}^{b}(n)=\operatorname{sym}_{1}(n) \in b^{\infty} .
$$

In order to calculate the one parameter groups of transformations corresponding to the generators (66), we have to solve the system of ordinary differential equations for the initial conditions $r(0)=r_{0}$ and $s(0)=s_{0}$ :

$$
\begin{aligned}
& r^{\prime}(\varepsilon)=e^{-r}|\lambda|\left(\Phi_{+}(\vec{x}, t) e^{|\lambda|\left(\frac{2 \nu_{2}}{\nu_{1}} r+s\right)}+\Phi_{-}(\vec{x}, t) e^{-|\lambda|\left(\frac{2 \nu_{2}}{\nu_{1}} r+s\right)}\right) \\
& s^{\prime}(\varepsilon)=e^{-r}\left(\left(1-\frac{2 \nu_{2}}{\nu_{1}}|\lambda|\right) \Phi_{-}(\vec{x}, t) e^{-|\lambda|\left(\frac{2 \nu_{2}}{\nu_{1}} r+s\right)}-\left(1+\frac{2 \nu_{2}}{\nu_{1}}|\lambda|\right) \Phi_{+}(\vec{x}, t) e^{|\lambda|\left(\frac{2 \nu_{2}}{\nu_{1}} r+s\right)}\right)
\end{aligned}
$$


We immediately deduce that

$$
\frac{d^{2}}{d \varepsilon^{2}} e^{2 r(\varepsilon)}=8 \Phi_{+}(\vec{x}, t) \Phi_{-}(\vec{x}, t) \lambda^{2}
$$

SO

$$
r(\varepsilon)=\frac{1}{2} \ln \left(4 \Phi_{+} \Phi_{-}|\lambda|^{2} \varepsilon^{2}+2 e^{r_{0}}|\lambda|\left(\Phi_{+} e^{\lambda\left(\frac{2 \nu_{2}}{\nu_{1}} r_{0}+s_{0}\right)}+\Phi_{-} e^{-\lambda\left(\frac{2 \nu_{2}}{\nu_{1}} r_{0}+s_{0}\right)}\right) \varepsilon+e^{2 r_{0}}\right) .
$$

Now $s(\varepsilon)$ is determined by equation (69), (The sign of the square root is fixed by the initial condition $s(0)=s_{0}$.)

$$
s(\varepsilon)=-\frac{2 \nu_{2}}{\nu_{1}} r(\varepsilon)+\frac{1}{|\lambda|} \ln \left( \pm \sqrt{\left(\frac{r^{\prime}(\varepsilon) e^{r(\varepsilon)}}{2|\lambda| \Phi_{+}}\right)^{2}-\frac{\Phi_{-}}{\Phi_{+}}}-\frac{r^{\prime}(\varepsilon) e^{r(\varepsilon)}}{2 \lambda \Phi_{+}}\right) .
$$

Together equations (72) and (73) constitute the one parameter group of transformations. Since $\Phi_{ \pm}$are solutions of a linear equation we can set $\varepsilon=\frac{1}{2}$ in these formulas without loss of generality and for every solution $\psi_{0}=\exp \left(r_{0}+i s_{0}\right)$ of the DG-equation equations (72) and (73) produce a new solution. In particular, if we start with the trivial solution $\psi_{0} \equiv 0$ of (9), i.e. $r_{0} \rightarrow-\infty$, we obtain a transformation mapping pairs of positive solutions $\left(\Phi_{+}, \Phi_{-}\right)$of the forward and backward heat equation to a solution of equation (9),

$$
\psi(\vec{x}, t)=\left(\Phi_{+}(\vec{x}, t) \Phi_{-}(\vec{x}, t)\right)^{\frac{1}{2}\left(1-i \frac{2 \nu_{2}}{\nu_{1}}\right)} \exp \left(\frac{i}{2|\lambda|} \ln \left(\frac{\Phi_{-}}{\Phi_{+}}\right)\right)
$$

It is in fact an extension of the transformation found by G. Auberson and P.C. Sabatier [9] for the special case $\nu_{2}=0$.

If on the other hand $\mu_{2}>2 \frac{\nu_{2}^{2}}{\nu_{1}}$, then $\lambda= \pm i \Lambda^{-1}, \Lambda=\sqrt{\frac{2 \nu_{1} \mu_{2}-4 \nu_{2}^{2}}{\nu_{1}^{2}}}$, is imaginary and we have to ensure that the coefficients of the vector-field are real valued. In this case we get the generator

$$
\begin{aligned}
Z_{\Psi}= & \frac{2}{\Lambda} e^{-r}|\Psi(\vec{x}, t)|\left(\sin \left(\frac{2 \nu_{2} r+\nu_{1} s}{\nu_{1} \Lambda}-\arg \Psi(\vec{x}, t)\right) \partial_{r}\right. \\
& \left.+\left(\Lambda \cos \left(\frac{2 \nu_{2} r+\nu_{1} s}{\nu_{1} \Lambda}-\arg \Psi(\vec{x}, t)\right)-\frac{2 \nu_{2}}{\nu_{1}} \sin \left(\frac{2 \nu_{2} r+\nu_{1} s}{\nu_{1} \Lambda}-\arg \Psi(\vec{x}, t)\right)\right) \partial_{s}\right)
\end{aligned}
$$

where $\Psi$ is a solution of the free linear SCHRÖDINGER equation

$$
i \partial_{t} \Psi=\nu_{1} \Lambda \Delta \Psi
$$

Again the algebra $c^{\infty}=\left\{Z_{\Psi} \mid \Psi\right.$ solution of (76) $\}$ is commutative, and the maximal LIE symmetry algebra is

$$
\operatorname{sym}_{1}^{c}(n)=\operatorname{sym}_{1}(n) \oplus c^{\infty} .
$$


$Z_{\Psi}$ can be integrated in analogy to the integration of $Z_{\Phi_{ \pm}}$above and we find the following solution:

$$
\begin{aligned}
r(\varepsilon)= & \frac{1}{2} \ln \left(4|\Psi|^{2} \frac{\varepsilon^{2}}{\Lambda^{2}}+4 e^{r_{0}}|\Psi| \sin \left(\Lambda^{-1}\left(\frac{2 \nu_{2}}{\nu_{1}} r_{0}+s_{0}\right)-\arg \Psi\right) \frac{\varepsilon}{\Lambda}+e^{2 r_{0}}\right), \\
s(\varepsilon)= & \Lambda \arg \Psi-\frac{\nu_{2}}{\nu_{1}} \ln \left(4|\Psi|^{2} \frac{\varepsilon^{2}}{\Lambda^{2}}+4 e^{r_{0}}|\Psi| \sin \left(\Lambda^{-1}\left(\frac{2 \nu_{2}}{\nu_{1}} r_{0}+s_{0}\right)-\arg \Psi\right) \frac{\varepsilon}{\Lambda}+e^{2 r_{0}}\right) \\
& +\Lambda \arcsin \left(\frac{2|\Psi| \frac{\varepsilon}{\Lambda}+e^{r_{0}} \sin \left(\Lambda^{-1}\left(\frac{2 \nu_{2}}{\nu_{1}} r_{0}+s_{0}\right)-\arg \Psi\right)}{\sqrt{4|\Psi|^{2} \frac{\varepsilon^{2}}{\Lambda^{2}}+4 e^{r_{0}}|\Psi| \sin \left(\Lambda^{-1}\left(\frac{2 \nu_{2}}{\nu_{1}} r_{0}+s_{0}\right)-\arg \Psi\right) \frac{\varepsilon}{\Lambda}+e^{2 r_{0}}}}\right) .
\end{aligned}
$$

Again we can set $\varepsilon=\frac{1}{2}$ without loss of generality and for every solution $\psi_{0}=\exp \left(r_{0}+i s_{0}\right)$ of the DG-equation (78) produces a new solution. Here, the trivial solution $\psi_{0} \equiv 0$ yields a linearizing transformation

$$
N_{(\Lambda, \gamma)}(\psi)=\psi^{\frac{1}{2}(1+\Lambda+i \gamma)} \bar{\psi}^{\frac{1}{2}(1-\Lambda+i \gamma)}=|\psi| e^{i(\gamma \ln |\psi|+\Lambda \arg \psi)}
$$

with $\gamma=-\frac{2 \nu_{2}}{\nu_{1}}$. We remark here that the definition (79) for non-integer $\Lambda$ is only formal due to the ambiguity of the phase $\arg \psi$. For integer values of $\Lambda$, however, it can be shown that the transformation is continuous in the $L^{2}$-topology [26].

\section{Gauge Classification}

In this section we first review some of the properties of the set of transformations $\mathcal{N}=$ $\left\{N_{(\Lambda, \gamma)} \mid(\Lambda, \gamma) \in \dot{R} \times \mathbb{R}\right\}$ that have been discussed in [10]. As the transformations (79) leave the probability density $\rho$ invariant, they were called nonlinear gauge transformations We will then apply the results to a gauge classification of the LIE symmetries of the DG-equation (9).

1. The set of these transformations is a realization of the affine group Aff(1) in one dimension

$$
N_{\left(\Lambda_{1}, \gamma_{1}\right)} \circ N_{\left(\Lambda_{2}, \gamma_{2}\right)}=N_{\left(\Lambda_{1} \Lambda_{2}, \Lambda_{1} \gamma_{2}+\gamma_{1}\right)}
$$

the inverse is therefore $N_{(\Lambda, \gamma)}^{-1}=N_{\left(\Lambda^{-1},-\Lambda^{-1} \gamma\right)}$.

2. The 8 parameter family (9) is invariant under this action of the affine group Aff (1), and the change of parameters is given by

$$
\begin{gathered}
\nu_{1}^{\prime}=\frac{\nu_{1}}{\Lambda}, \quad \nu_{2}^{\prime}=-\frac{\gamma}{2 \Lambda} \nu_{1}+\nu_{2}, \\
\mu_{1}^{\prime}=-\frac{\gamma}{\Lambda} \nu_{1}+\mu_{1}, \quad \mu_{2}^{\prime}=\frac{\gamma^{2}}{2 \Lambda} \nu_{1}-\gamma \nu_{2}-\frac{\gamma}{2} \mu_{1}+\Lambda \mu_{2}, \quad \mu_{3}^{\prime}=\frac{\mu_{3}}{\Lambda} \\
\mu_{4}^{\prime}=-\frac{\gamma}{\Lambda} \mu_{3}+\mu_{4}, \quad \mu_{5}^{\prime}=\frac{\gamma^{2}}{4 \Lambda} \mu_{3}-\frac{\gamma}{2} \mu_{4}+\Lambda \mu_{5}, \quad \mu_{0}^{\prime}=\Lambda \mu_{0} .
\end{gathered}
$$


3. The concept of a gauge classification of the DG-equation can be taken further. Since we have an action of a two dimensional group on an eight dimensional space of parameters, an appropriate description of the family (7) is by six parameters invariant under the group action, and two gauge parameters. A possible choice of gauge invariants is:

$$
\begin{gathered}
\iota_{1}=\nu_{1} \mu_{2}-\nu_{2} \mu_{1}, \quad \iota_{2}=\mu_{1}-2 \nu_{2}, \quad \iota_{3}=1+\mu_{3} / \nu_{1}, \quad \iota_{4}=\mu_{4}-\mu_{1} \mu_{3} / \nu_{1}, \\
\iota_{5}=\nu_{1}\left(\mu_{2}+2 \mu_{5}\right)-\nu_{2}\left(\mu_{1}+2 \mu_{4}\right)+2 \nu_{2}^{2} \mu_{3} / \nu_{1}, \quad \iota_{0}=\nu_{1} \mu_{0} .
\end{gathered}
$$

Thus, for the free equation the five parameters $\iota_{1}, \ldots, \iota_{5}$ determine an equivalence class of nonlinear Schrödinger equations.

First we remark that the gauge transformations explain the modifications in the representation of the SCHRÖDINGER group found in section 2.2: The transformation of the parameters (81) shows that there is always at least one nonlinear gauge transformation such that $\mu_{1}=0$. So the modified projective transformations and dilations (54) for $\mu_{1} \neq 0$ are related to the usual ones $\left(\mu_{1}=0\right)$ by a nonlinear gauge transformation $N_{\left(1, \mu_{1} / \nu_{1}\right)}$.

Furthermore, the conditions for the various maximal LIE symmetries of the DG-equation have to be invariant under $\mathcal{N}$ and we can characterize the corresponding subfamilies by gauge invariants $\iota_{j}$. Summarizing the results of section 2 and the relations between the various subfamilies we obtain the following picture:

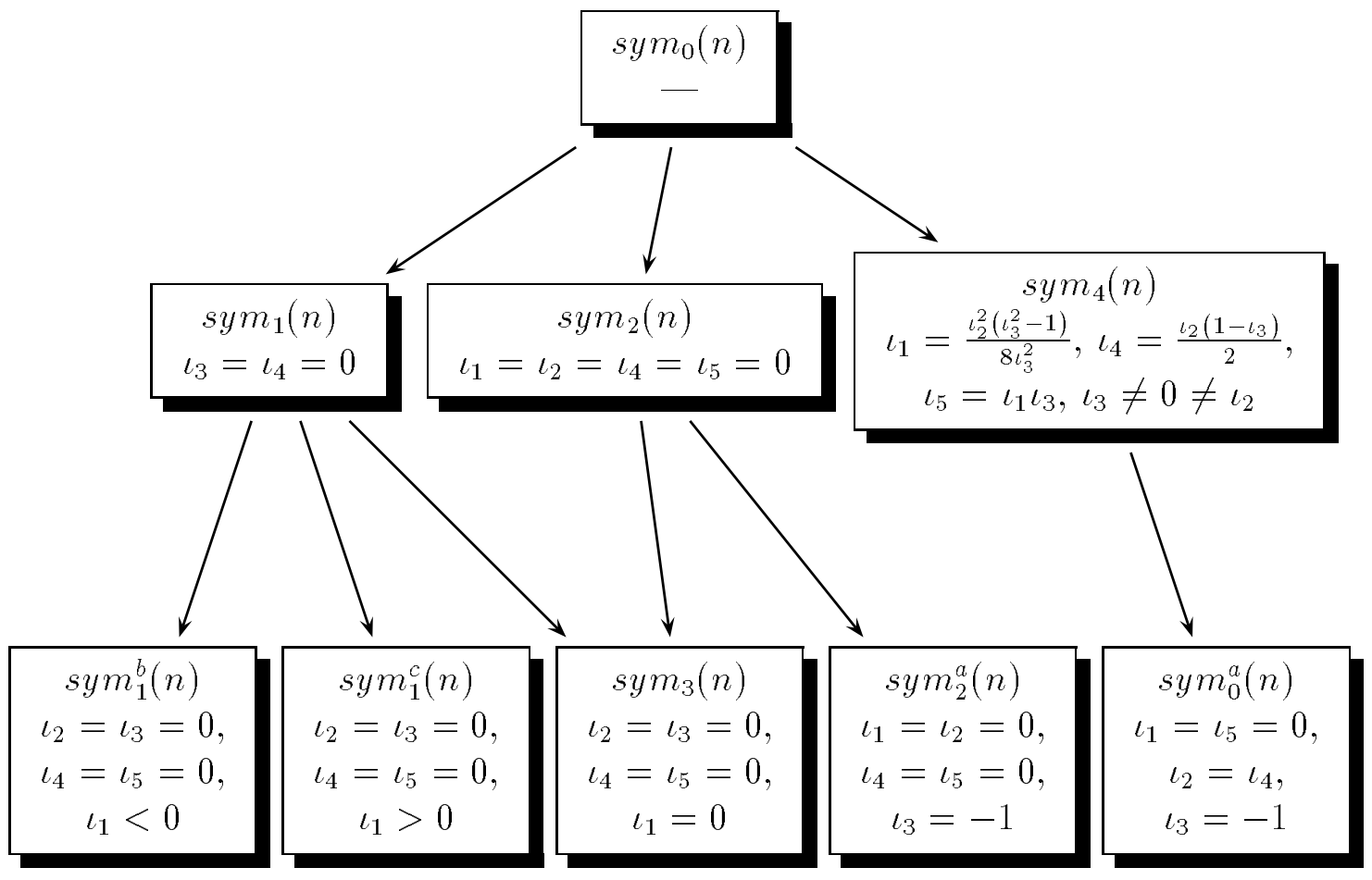

Fig. 1: LIE symmetries of the DG-equation. Subfamilies are characterized by gauge invariants and arrows indicate the subfamily-structure. 


\section{Conclusions}

In this paper we have systematically discussed the LIE symmetry of the $n+1$-dimensional DG-equation (7) in a convenient parameterization (9). Apart from the generators of the SCHRÖDINGER group and complex homogeneity, we have found two new generators $A$ and $F$ giving rise to the finite dimensional LIE symmetry algebras $\operatorname{sym}_{2}(n), \operatorname{sym}_{3}(n)$ and $\operatorname{sym}_{4}(n)$. Furthermore we obtained four different types of infinite dimensional LIE symmetry algebras, $\operatorname{sym}_{0}^{a}(n), \operatorname{sym}_{2}^{a}(n), \operatorname{sym}_{1}^{b}(n)$, and $\operatorname{sym}_{1}^{c}(n)$. The last two correspond to a local linearization of a subfamily of the DG-equation to a forward and backward heat equation, and a linear SCHRÖDINGER equation, respectively. Due to the systematic approach there are no other local linearizations of the DG-equation.

One type of linearizing transformations leaves the whole family of equations invariant, and was used to classify the subfamilies of the various LIE symmetry algebras by gauge invariant parameters $\iota_{j}$ (Fig 1.). The subfamily $\iota_{2}=\ldots=\iota_{5}=0, \iota_{1} \neq 0$, is outstanding because of its linearizability. The limiting case $\iota_{1}=0$ can be transformed into EuLERs equation [9] and the methods to solve this subfamily can be adapted to the subfamily $\iota_{1}=\iota_{2}=\iota_{4}=\iota_{5}=0$ [27. The next candidate for a solvable subfamily seems to be the subfamily $\iota_{1}=\iota_{5}=0$, $\iota_{2}=\iota_{4}, \iota_{3}=-1$, where the infinite dimensional LIE symmetry algebra might be used to obtain a general solution. We postpone this for a future paper.

The results for the SCHRÖDINGER invariant subfamily (38) are consistent (up to a nonlinear gauge transformation) with the results of G. RideAu and P. Winternitz [25] in $1+1$ dimensions, who classified all evolution equations with $s c h_{e}(1)$ LIE symmetry algebra up to second order in spatial derivatives. The family (38) in the gauge $\nu_{1}=-1$ and $\mu_{1}=0$ is contained in their equation (3.6) for the special choice $S(u, v)=\left(-i \nu_{2}+\mu_{2}+\mu_{5}-\frac{1}{4}\right) u^{2}+\left(i \nu_{2}-\right.$ $\left.\mu_{2}+\frac{1}{2}\right) v$.

Finally note that for the subfamily (64) there exists a HAMILTON functional generating the evolution equation [5, 10] and the corresponding DG-equation fits into the scheme of a nonlinear quantum theory proposed by S. WEINBERG 28. Furthermore a consistent concept of 'nonlinear' observables (different from the one proposed by S. WEINBERG) can be developed

rigorously for the particular subfamily $\mu_{2}=\frac{1}{2} \nu_{1}+2 \frac{\nu_{2}^{2}}{\nu_{1}}$ of (64) establishing full equivalence between this model and linear quantum mechanics [26].

\section{Acknowledgments}

I would like to thank H.-D. Doebner, W. Scherer, and R. ZhDAnov for useful discussions. I am also grateful to the organizers of the XXVIIth Symposium on Mathematical Physics in Torun for their hospitality.

\section{References}


[1] H.-D. Doebner and G.A. Goldin: "On a general nonlinear Schrödinger equation admitting diffusion currents". Phys. Lett. A 162, 397-401 (1992).

[2] H.-D. Doebner and G.A. Goldin: "Manifolds, general symmetries, quantization and nonlinear quantum mechanics". in Proceedings of the First German-Polish Symposium on Particles and Fields, Rydzyna Castle, 1992, p. 115 World Scientific, Singapore, 1993.

[3] H.-D. Doebner and G.A. Goldin: "Group theoretical foundations of nonlinear quantum mechanics". in Annales de Fisica, Monografias, Vol. II, Proceedings of the 17th International Conference on Group Theoretical Methods in Physics, Salamanca, 1992, p. 442-445 CIEMAT, Madrid, 1993.

[4] H.-D. Doebner and G.A. Goldin: "Properties of nonlinear Schrödinger equations associated with diffeomorphism group representations". J. Phys. A: Math. Gen. 27, 17711780 (1994).

[5] P. Nattermann: "Struktur und Eigenschaften einer Familie nichtlinearer Schrödingergleichungen". Diplomarbeit, Technische Universität Clausthal, August 1993.

[6] H.-D. Doebner and J. D. Hennig: "A quantummechanical evolution equation for mixed states from symmetry and kinematics". Clausthal-preprint ASI-TPA/1/95, 1995.

[7] G. Lindblad: "On the generators of quantum dynamical semigroups". Comm. Math. Phys. 48, 110-130 (1976).

[8] P. Nattermann: "Solutions of the general Doebner-Goldin equation via nonlinear transformations". in Proceedings of the XXVI Symposium on Mathematical Physics, Torun, December 7-10, 1993, p. 47-54 Nicolas Copernicus University Press, Toruń, 1994.

[9] G. Auberson and P.C. Sabatier: "On a class of homogemeous nonlinear Schrödinger equations". J. Math. Phys. 35, 4028-4040 (1994).

[10] H.-D. Doebner, G.A. Goldin, and P. Nattermann: "A family of nonlinear Schrödinger equations: Linearizing transformations and resulting structure". Clausthalpreprint ASI-TPA/8/94, to appear in Proceedings of the XIIIth Workshop on Mathematical Methods in Physics, Bialowieza, July 9-15, 1994,c Plenum Press, 1995.

[11] G.A. Goldin: "The diffeomorphism group appoach to nonlinear quantum systems". Int. J. Mod. Phys. B 6, 1905-1916 (1992).

[12] V.V. Dodonov and S.S. Mizrahi: "Doebner-Goldin nonlinear model of quantum mechanics for a damped oscillator in a magnetic field". Phys. Lett. A 181, 129-134 (1993).

[13] P. Nattermann, W. Scherer, and A.G. Ushveridze: "Exact solutions of the general Doebner-Goldin equation". Phys. Lett. A 184, 234-240 (1994). 
[14] P. Nattermann, W. Scherer, and A.G. Ushveridze: "Correlated coherent states of the general Doebner-Goldin equation". to appear in Int. J. Mod. Phys. B, 1995.

[15] G.A. Goldin, B.A. Malomed, and L. Stenflo: "Modulational stability of plane waves for a class of nonlinear Schrödinger equations". to appear in Nonlinear, Deformed, and Irreversible Quantum Systems, proceedings of the International Symposium "Nonlinear, Dissipative, Irreversible Quantum Systems", Clausthal, August 15-19 1994, World Scientific, Singapore, 1995.

[16] P.C. SABATIER: "Multidimensional nonlinear Schrödinger equations with exponentially confined solutions", Inverse Problems 6, L47-L53 (1990).

[17] P. Nattermann: "Maximal Lie symmetry of the free general Doebner-Goldin equation in 1+1 dimensions". Clausthal-preprint ASI-TPA/9/94, 1994.

[18] U. NIEDERER: "The maximal kinematical invariance group of the free Schrödinger equation". Helv. Phys. Acta 45, 802-810 (1972).

[19] C.R. Hagen: "Scale and conformal transformations in galilean-covariant field theory" . Phys. Rev. D 5, 377-388 (1972).

[20] A.O. Barut and Bo Wei Xu: "Conformal covariance and the probability interpretation of wave equations". Phys. Lett. A 82, 218-220 (1981).

[21] L.V. Ovsiannikov: Group Analysis of Differential Equations. Academic Press, New York, 1982.

[22] P.J. Olver: Appplications of Lie Groups to Differential Equations, volume 107 of Graduate Texts in Mathematics. Springer Verlag, Heidelberg, 1986.

[23] W.I. Fushchych, V.M. Shtelen, and N.I. Serov: Symmetry Analysis and Exact Solutions of Equations of Nonlinear Mathematical Physics. Kluwer Academic Publishers, Dordrecht, 1993.

[24] G. Baumann: "Lie symmetries of differential equations: A Mathematica program to determine Lie symmetries", (Wolfram Research Inc., Champaign, Ilinois, MathSource 0202-622 1992).

[25] G. Rideau and P. Winternitz: "Evolution equations invariant under two-dimensional space-time Schrödinger group". J. Math. Phys. 34, 558-570 (1993).

[26] W. LüCKE: "Nonlinear Schrödinger dynamics and nonlinear observables". to appear in Nonlinear, Deformed, and Irreversible Quantum Systems, proceedings of the International Symposium "Nonlinear, Dissipative, Irreversible Quantum Systems", Clausthal, August 15-19 1994, World Scientific, Singapore, 1995. 
[27] R. ZhDANOv: private communication

[28] S. Weinberg: "Testing quantum mechanics". Ann. Phys. (NY) 194, 336-386 (1989). 